\title{
Positive moods can eliminate intentional forgetting
}

\author{
Karl-Heinz BäUmL and Christof Kuhbandner \\ Regensburg University, Regensburg, Germany
}

\begin{abstract}
People can intentionally forget previously studied information if, after study, a forget cue and new material to be encoded are provided. We examined how the affective state people experience during encoding of the new material modulates such directed forgetting. Positive, negative, and neutral moods were induced immediately before the new material was studied. The study materials themselves were neutral. The results showed sustained forgetting of the previously studied materials in negative moods but an elimination of the forgetting in positive moods. These findings agree with the effects of mood found for other cognitive tasks. They suggest that in positive moods, associative networks are activated, which leads to reactivation of List-1 items, and thus to elimination of the directed forgetting effects. These results contrast with recent reports on the role of emotional content in directed forgetting, which have described equivalent effects for neutral and emotional materials. Together, our findings suggest that directed forgetting is mainly affected by mood, and hardly at all by emotional content.
\end{abstract}

Affective states can influence cognition. Such influences have been observed across a wide range of tasks, including tasks involving attention, reasoning, and problem solving. For instance, people in positive moods have been reported to process incoming information in a much more global manner than do those in negative moods (Gasper $\&$ Clore, 2002). People in positive moods also seem more likely than those in negative moods to use judgment heuristics (Schwarz, Bless, \& Bohner, 1991; for a review, see Clore \& Huntsinger, 2007).

Affective states can also influence memory performance, and the effects can again be different for positive and negative moods. For instance, individuals in positive moods have been reported to show stronger priming effects (Hänze \& Hesse, 1993), produce more unusual associations (Isen, Johnson, Mertz, \& Robinson, 1985), and more easily find connections between weakly related words (Bolte, Goschke, \& Kuhl, 2003) than individuals in negative moods. In addition, participants in positive moods have been reported to show higher levels of false memories than do participants in negative moods (Storbeck \& Clore, 2005). Overall, positive, but not negative, moods seem to promote the activation of widespread associative networks in human memory (Clore \& Huntsinger, 2007).

\section{Directed Forgetting}

Listwise directed forgetting is a form of intentional forgetting in which the accessibility of irrelevant, out-ofdate information is reduced and retrieval of more relevant, newer information is selectively enhanced. In this paradigm, subjects study two lists of items and, after presenta- tion of List 1, receive a cue to either forget or continue remembering the list before studying List 2 . When later asked to recall all of the previously presented items, including those they were originally cued to forget, forgetcued subjects typically show impaired recall of List 1 and improved recall of List 2, reflecting reduced accessibility of the old, out-of-date information and improved access to the more relevant, newer information (for reviews, see MacLeod, 1998, or Bäuml, 2008).

Directed forgetting has mostly been attributed to a single mechanism, regarded as responsible for both effects of the forget cue - that is, List-2 enhancement and List-1 forgetting. The selective rehearsal account, for instance, assumes that during List-2 encoding, subjects in the remember condition rehearse both the List- 2 and the to-be-remembered List-1 items, whereas in the forget condition the forget cue leads to selective rehearsal activities on the List- 2 items, thus improving later recall of List 2 at the expense of List 1 (R. A. Bjork, 1970). The retrieval inhibition account assumes that by inhibiting List-1 items, the forget cue reduces accessibility of those items and, because of the resulting decrease in the items' interference potential, simultaneously improves access to the List-2 items (Geiselman, Bjork, \& Fishman, 1983). Finally, the context change account claims that the forget cue induces a change in subjects' internal context; such a context change should impair List-1 recall, because of the mismatch between the contexts at encoding and retrieval, and improve List-2 recall, because of reduced interference (Sahakyan \& Kelley, 2002; for two-mechanism accounts of directed forgetting, see Bäuml, Hanslmayr, Pastötter, \& Klimesch, 2008; Sahakyan \& Delaney, 2003).

K.-H. Bäuml, karl-heinz.baeuml@psychologie.uni-regensburg.de 


\section{Mood and Directed Forgetting}

When addressing the role of emotion in directed forgetting, two related but separate questions arise. The first is whether directed forgetting differs for emotional and neutral List-1 contents, independently of a subject's current affective state when trying to forget. The other is whether the affective state experienced when trying to forget influences List-1 forgetting, independently of the List-1 contents. The goal of the research we report here was to address the second question by investigating how affective states might influence directed forgetting.

On the basis of the finding that positive, but not negative, moods promote the activation of associative networks (Bolte et al., 2003; Hänze \& Hesse, 1993; Storbeck \& Clore, 2005), it may be hypothesized that positive, but not negative, moods can eliminate the effects of directed forgetting. Indeed, from the prior work, in the forget condition the promotion of associative activation in positive moods may lead to activation of List-1 items during List-2 encoding. Such activation of List-1 items may undo the effect of the forget cue and eliminate both List-1 forgetting and List- 2 enhancement. In fact, according to the selective rehearsal account (R. A. Bjork, 1970), reactivation of List-1 items during List-2 encoding could remind participants of List-1 items, thereby reversing the consequences of List-1 rehearsal termination; with retrieval inhibition (Geiselman et al., 1983), reactivation of List-1 items could induce a release from inhibition (Basden, Basden, \& Wright, 2003; E. L. Bjork \& Bjork, 1996); and in the context change account (Sahakyan \& Kelley, 2002), reactivation of List-1 items could reinstate the original List-1 context, thereby reducing contextual differentiation between the two lists. In contrast to positive moods, negative moods are not supposed to promote activation of associative networks (Clore \& Huntsinger, 2007). As a result, no comparable reactivation of List-1 items during List-2 encoding should take place, and the directed forgetting effects should not be eliminated.

Sahakyan and Goodmon (2007) recently showed that directed forgetting can be eliminated by varying study content. Indeed, in their study, when each List-1 item had a bidirectionally related item on List 2, neither List-1 forgetting nor List-2 enhancement was observed (for a related result, see Conway, Harries, Noyes, Racsma'ny, \& Frankish, 2000). The authors argued that, in the presence of bidirectional associations, the backward associations may allow List-2 encoding to reactivate List-1 items, thus undoing the effect of the forget cue and eliminating List-1 forgetting. The present study examines whether reactivation of List-1 items can also be created by mood.

\section{The Present Experiment}

To the best of our knowledge, this study is the first to examine the role of affective states in directed forgetting. Our subjects studied two lists that had to be recalled on a later memory test. Between the study of the two lists, subjects received either a remember cue, indicating they should remember the first list, or a forget cue, indicating they should forget that list. Immediately before List 2 was presented, positive, negative, or neutral moods were induced. In line with prior work on the role of mood in cognition, we hypothesized that positive moods during List-2 encoding lead to the reactivation of List-1 items, and thus eliminate both List-1 forgetting and List-2 enhancement. Negative moods should not trigger such processes, and thus should leave the two directed forgetting effects largely unaffected.

\section{METHOD}

\section{Subjects}

Seventy-two students at Regensburg University participated in the experiment. Twenty-four were randomly assigned to each of three mood conditions (positive, negative, or neutral).

\section{Materials}

Four word lists were constructed, each consisting of 15 unrelated neutral nouns of medium frequency. The words were selected from the CELEX database using the WordGen software toolbox (version 1.0; Duyck, Desmet, Verbeke, \& Brysbaert, 2004). Across lists, the words were matched on frequency and word length. Each list was used equally often in each experimental condition and served equally often as the list presented first or second in each condition.

Sets of 10 positive, 10 negative, and 10 neutral pictures were selected from the International Affective Picture System (IAPS; Lang, Bradley, \& Cuthbert, 1999), a series of pictures with standardized ratings for valence and arousal. ${ }^{1}$ The pictures were selected according to their ratings for valence in the technical report of the IAPS (positive, $M=7.6$; negative, $M=2.3$; neutral, $M=5.0)[F(2,27)=$ $\left.172.5, M S_{\mathrm{e}}=0.382, p<.001, \eta^{2}=.93\right]$, with the restriction that positive and negative pictures were matched for their arousal ratings (positive, $M=5.6$; negative, $M=6.4$ ) $\left[F(1,9)=4.3, M S_{\mathrm{e}}=0.885\right.$, $p>.05]$. Arousal for the emotional pictures differed from that of the neutral pictures $(M=2.8)\left[F(1,9)=641.8, M S_{\mathrm{e}}=0.105, p<\right.$ $.001]$. Positive and negative pictures of the IAPS can effectively induce emotions (Bäuml \& Kuhbandner, 2007). To assess the success of emotion induction in the present experiment, we used the affect grid (Russell, Weiss, \& Mendelsohn, 1989), which permits subjects to express their emotional experience on a $9 \times 9$ matrix varying in the dimensions of valence and arousal. Along both dimensions, the scales range from 1 (extremely negative, low arousal) to 9 (extremely positive, high arousal).

\section{Design}

We used a $3 \times 2$ mixed design with the between-subjects factor of mood (positive, negative, neutral) and the within-subjects factor of cue (remember, forget). For each subject, the experiment consisted of two parts, separated by a 5-min break. In each of the parts, two lists were presented for study and had to be recalled. The two parts differed only in the cue that subjects received between the learning of the two lists. In the remember condition, a remember cue indicated that the items of List 1 should be remembered; in the forget condition, a forget cue indicated that List 1 should be forgotten. The order of conditions was counterbalanced across subjects. The three mood conditions differed only in the type of mood that was induced before List 2 was presented for study.

\section{Procedure}

Subjects were informed that they should learn several word lists in this experiment, but that only some of the learned lists would be tested later. After presentation of a list, the experimenter would declare whether a list would be tested or whether it would no longer be relevant and should therefore be forgotten (Bäuml et al., 2008; Zellner \& Bäuml, 2006). Then, the List-1 items for the first part of the experiment were displayed on a computer screen in a random order. Each item was shown for $4.5 \mathrm{sec}$, with an interstimulus interval of $0.5 \mathrm{sec}$. Immediately after the list's last word was presented, the interlist cue was provided. Subjects were presented with either a 
remember cue, stating that the prior list should be remembered for a later test, or a forget cue, stating that the prior list would not be tested later and the list should be forgotten. After cue presentation, mood induction followed. In each of the three mood conditions, subjects were successively shown five pictures of the same valence and were told to let the pictures influence their emotional state. Each picture was presented for $6 \mathrm{sec}$.

Then, List 2 was presented for study, using the same procedure as for the List-1 study phase, followed by a remember cue telling subjects that List 2 should be remembered. After cue presentation, subjects were provided with the affect grid, on which they should mark the square that best represented their current mood state. A distractor phase followed, in which the subjects worked through a simple reaction time task for $2.5 \mathrm{~min}$. In the test phase, both studied lists had to be recalled, regardless of whether List 1 had been cued to be remembered or forgotten. Subjects in the forget condition were explicitly told that the forget cue received during study was only a pretense. Because the focus of the study was on recall of the first list, List 1 was always tested first (Sahakyan \& Kelley, 2002; Zellner \& Bäuml, 2006). Subjects were asked to freely recall a list's words in any order they wished. Recall of the two lists was carried out on two separate sheets of paper, and the subjects were given at least $1 \mathrm{~min}$ to write down as many words of a list as possible. After a 5-min break, the second part of the experiment started. With the exception of the cue condition (forget or remember) and new sets of words and pictures, this second part was identical to the first part of the experiment.

\section{RESULTS}

\section{Mood Manipulation Check}

Across conditions, subjects varied reliably in mood, as indicated by the valence measure (positive, $M=6.2$; negative, $M=5.1$; neutral, $M=5.9)\left[F(2,69)=5.2, M S_{\mathrm{e}}=\right.$ $\left.1.537, p<.01, \eta^{2}=.13\right]$. Planned comparisons revealed that valence in the negative mood differed reliably from valence in the positive and neutral moods (both $p \mathrm{~s}<.05$ ), whereas valence in the positive and neutral moods did not vary significantly $[F(1,46)<1]$. As indicated by the arousal measure, subjects did not differ in arousal level across the conditions (positive, $M=6.4$; negative, $M=6.4$; neutral, $M=6.0)[F(2,69)<1]$. Neither the valence nor the arousal measures varied significantly between subjects' first and second parts of the experiment (all $p \mathrm{~s}>.15$ ).

\section{Recall Test}

For all analyses, a list's percentage of correctly recalled words was the dependent measure. A word was counted as correctly recalled if it was named on the corresponding sheet of paper on which the test of a list was carried out. The mean rate of List- 2 intrusions during List-1 recall was below $5 \%$; it did not vary with cue or mood (all $F \mathrm{~s}<1$ ). The mean rate of List- 1 intrusions during List- 2 recall showed the same pattern (all $F \mathrm{~s}<1$ ).

Recall rates are shown in Figure 1. List-1 forgetting and List-2 enhancement were analyzed separately. Regarding List-1 forgetting, a 2 (cue: remember, forget) $\times 3$ (mood: positive, negative, neutral) ANOVA revealed no main effect of $\operatorname{mood}\left[F(2,69)=1.0, M S_{\mathrm{e}}=.069, p>.35\right]$ but a significant main effect of cue $\left[F(1,69)=12.9, M S_{\mathrm{e}}=\right.$ $\left..022, p<.001, \eta^{2}=.16\right]$, reflecting higher overall recall in the remember condition than in the forget condition, and a marginally significant interaction between the two factors $\left[F(2,69)=2.8, M S_{\mathrm{e}}=.022, p=.06, \eta^{2}=.08\right]$. Planned comparisons showed that significant List-1 forgetting arose in neutral $\left[F(1,23)=9.6, M S_{\mathrm{e}}=.020, p<\right.$ $\left..01, \eta^{2}=.29\right]$ and negative $\left[F(1,23)=8.2, M S_{\mathrm{e}}=.025\right.$, $\left.p<.01, \eta^{2}=.26\right]$ moods, but did not arise in positive moods $[F(1,23)<1]$.

Regarding List- 2 enhancement, a 2 (cue: remember, forget) $\times 3$ (mood: positive, negative, neutral) ANOVA revealed a significant main effect of cue $[F(1,69)=13.1$, $\left.M S_{\mathrm{e}}=.028, p<.001, \eta^{2}=.16\right]$, reflecting higher overall recall in the forget condition than in the remember condi-
List 1

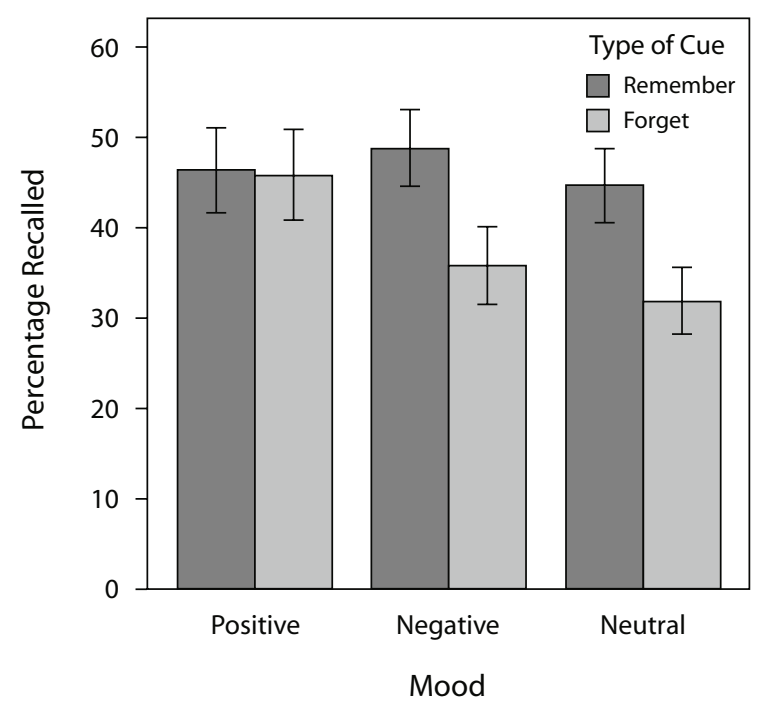

List 2

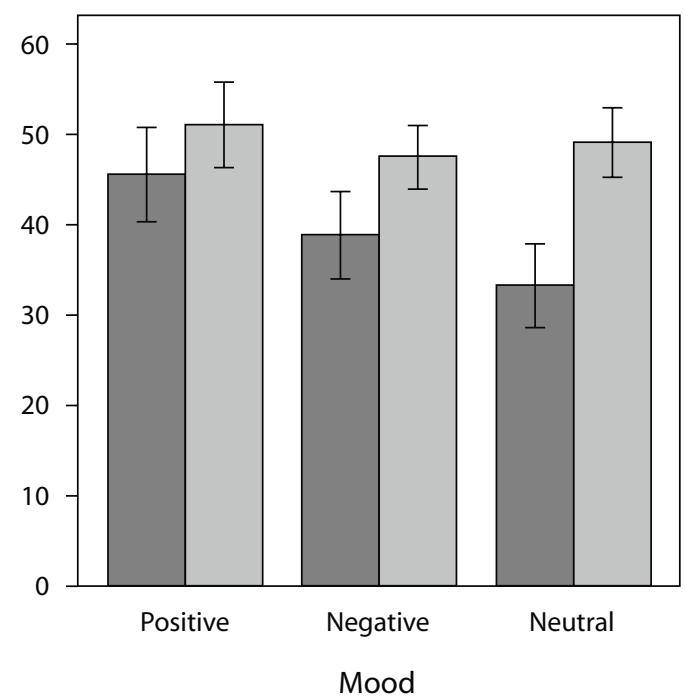

Figure 1. Recall of List-1 and List-2 items as a function of mood (positive vs. negative vs. neutral) and cue (remember vs. forget). The error bars represent standard errors. 
tion, but no significant main effect of mood $[F(2,69)<1]$ and no significant interaction between the two factors $\left[F(2,69)=1.2, M S_{\mathrm{e}}=.028, p=.30\right]$. Planned comparisons showed that significant List-2 enhancement arose in neutral moods $\left[F(1,23)=12.9, M S_{\mathrm{e}}=.023, p<.01\right.$, $\left.\eta^{2}=.36\right]$, but not in negative $\left[F(1,23)=2.7, M S_{\mathrm{e}}=.033\right.$, $p=.11]$ or in positive $\left[F(1,23)=1.4, M S_{\mathrm{e}}=.026, p=\right.$ .25] moods.

\section{Analysis of Possible Order Effects}

The present experiment used a within-subjects design, with each subject participating in both the remember and forget conditions. Our results replicate prior work reporting no effect of order of the remember and forget conditions on subjects' List-1 forgetting or List-2 enhancement (Bäuml et al., 2008; Zellner \& Bäuml, 2006). We found the same amount of forgetting $[F(1,70)<1]$ and the same amount of enhancement $\left[F(1,70)=2.2, M S_{\mathrm{e}}=.027, p=\right.$ .14] regardless of whether the subjects started with the remember or the forget condition. There was also no reliable interaction between order and $\operatorname{mood}($ all $F \mathrm{~s}<1)$.

\section{DISCUSSION}

Affective states can influence directed forgetting. When a neutral or negative mood was induced during List-2 encoding, the typical impairment of List-1 recall in the presence of the forget cue arose. In contrast, when a positive mood was induced during List-2 encoding, the forgetting of List-1 items was eliminated. Regarding List 2, reliable enhancement in response to the forget cue arose only when subjects were in a neutral mood during List-2 encoding, not when they were in a positive or negative mood, although the amount of enhancement did not vary reliably across the three mood conditions.

\section{Effects of Positive and Negative Moods}

These results are consistent with the view that the promotion of associative activation in positive moods (Clore \& Huntsinger, 2007) can lead to activation of to-beforgotten List-1 items during List-2 encoding, thus undoing the effect of the forget cue and eliminating both List-1 forgetting and List- 2 enhancement. This finding mimics a recent result on the role of study content in directed forgetting, showing that, if each List-1 item has a bidirectionally related item on List 2, both List-1 forgetting and List-2 enhancement disappear (Sahakyan \& Goodmon, 2007). The present finding also mimics the findings of previous work on the effects of partial reexposure of List-1 items on directed forgetting. Several studies have shown that, after List-2 encoding, reexposure of a subset of List-1 items can undo the effect of the forget cue and eliminate both List-1 forgetting and List-2 enhancement (Basden et al., 2003; E. L. Bjork \& Bjork, 1996). Together with the present work, these studies suggest that reactivation of List-1 items after presentation of the forget cue - whether as a result of positive mood, reexposure of the items, or content-can reduce, or even eliminate, directed forgetting effects, thus establishing an important boundary condition for this type of intentional forgetting.
In contrast to positive moods, negative moods are generally not assumed to promote associative activation processes (Clore \& Huntsinger, 2007). In the forget condition, List-2 encoding thus was not expected to trigger List-1 activation when combined with a negative mood, and neither List-1 forgetting nor List-2 enhancement should have been eliminated. Our finding of List-1 forgetting for negative moods is consistent with this prediction. Although no reliable List-2 enhancement was found in a negative mood, the amount of enhancement did not differ reliably from that in a neutral mood. Thus, the results for negative mood, like the results for positive mood, largely agree with expectation. In a recent study, we reported a neural signature of List-1 forgetting measured during List-2 encoding (Bäuml et al., 2008). To further pin down the mechanisms operating during a positive mood, future work should examine whether positive, but not negative, moods affect this neural signature and make it disappear.

The present results suggest an effect of positive mood on directed forgetting, although in the mood manipulation check the difference in valence ratings between the positive and neutral mood states was not reliable. The subjects' mood states were measured after List- 2 encoding, and thus not directly after mood induction. This procedure was used because the effect of the presentation of emotional pictures is known to fade with time (Garrett \& Maddock, 2001), and List-2 encoding therefore needed to follow the mood induction phase as soon as possible. Several studies with mood induction procedures similar to the one in the present study have shown that, directly after mood induction, substantial changes in the experienced mood state could be measured, whereas after completion of the cognitive task, mood induction effects were often small, if they existed at all (Eich \& Metcalfe, 1989). Still, numerous findings have indicated that the presentation of positive pictures is a highly effective method to induce positive emotions (e.g., Bradley, Cuthbert, \& Lang, 1996).

\section{Associative Activation Versus Impaired Executive Functioning}

The present finding that positive, but not negative, moods eliminate the effects of directed forgetting agrees with the view that positive moods lead to the activation of associative networks and thus undo the effect of a forget cue. The present effects of mood on directed forgetting may be explained in an alternative way, however. This explanation rests on the view that directed forgetting depends on executive functioning (Conway et al., 2000; Delaney \& Sahakyan, 2007), in combination with results showing that positive, but not negative, moods can impair such functioning (for a review, see Mitchell \& Phillips, 2007). However, reduced executive functioning is often assumed to reduce recall levels (Conway et al., 2000), and our finding in the present experiment of no such reduction in List-2 recall levels in positive moods may be regarded as a challenge for this explanation. Moreover, the associative activation account provides the more parsimonious explanation of the present results, because it can account for the influence of affective states not only in directed forgetting but 
also in other episodic memory paradigms, such as the false memory paradigm (Storbeck \& Clore, 2005).

\section{Valence Versus Arousal}

Recent debate in the literature has focused on whether valence or arousal contributes to mood differences in processing style. On the basis of findings on the role of mood in false memories, some have argued that valence (Storbeck \& Clore, 2005), and others that arousal (Corson \& Verrier, 2007), is key. In the present study, List-1 forgetting was eliminated in positive moods but was unaffected in negative moods. The fact that valence, and not arousal, differed between the two mood conditions suggests that it was likely valence, not arousal, that caused the processing differences in the present paradigm. A similar indication was found in a recent study on the role of mood in retrieval-induced forgetting (Bäuml \& Kuhbandner, 2007).

\section{Emotional Content Versus Affective State}

The present study addressed the question of whether an affective state induced when people try to forget influences List-1 forgetting, independent of the valence of List-1 contents. In contrast, prior work addressed the question of whether List-1 forgetting differs for emotional and neutral List-1 contents, independent of people's affective state when they try to forget. Although, for individuals with acute stress disorder, greater List- 1 forgetting has been reported for positive and negative materials than for neutral materials (Moulds \& Bryant, 2005), in general, hardly any effects of the materials on List-1 forgetting have been found to date (Barnier et al., 2007; McNally, Clancy, Barrett, \& Parker, 2004). The effect of emotion on directed forgetting thus seems to be largely restricted to the affective states people experience when they try to forget.

\section{AUTHOR NOTE}

The research reported here was supported by a grant from the Deutsche Forschungsgemeinschaft to K.-H.B. (Ba 1382/7-1). We thank L. Sahakyan and the anonymous referees for their comments on a previous version of the manuscript, and O. Kliegl and L. Knoll for their help with data collection. Correspondence concerning this article should be addressed to K.-H. Bäuml, Department of Experimental Psychology, Regensburg University, 93040 Regensburg, Germany (e-mail: karl-heinz .baeuml@psychologie.uni-regensburg.de).

\section{REFERENCES}

Barnier, A. J., Conway, M. A., Mayoh, L., SPeyer, J., Avizmil, O., \& HARRIS, C. B. (2007). Directed forgetting of recently recalled autobiographical memories. Journal of Experimental Psychology: General, 136, 301-322.

Basden, B. H., Basden, D. R., \& Wright, M. J. (2003). Part-list reexposure and release of retrieval inhibition. Consciousness \& Cognition, 12, 354-375.

BäUML, K.-H. (2008). Inhibitory processes. In J. H. Byrne (Series Ed.) \& H. L. Roediger III (Vol. Ed.), Learning and memory: A comprehensive reference. Vol. 2: Cognitive psychology of memory (pp. 195-220). Amsterdam: Elsevier.

BäUml, K.-H., Hanslmayr, S., Pastötter, B., \& Klimesch, W. (2008). Oscillatory correlates of intentional updating in episodic memory. NeuroImage, 41, 596-604.

BäUmL, K.-H., \& Kuhbandner, C. (2007). Remembering can cause forgetting-but not in negative moods. Psychological Science, 18, 111-115.
BJork, E. L., \& BJoRK, R. A. (1996). Continuing influences of to-beforgotten information. Consciousness \& Cognition, 5, 176-196.

BJork, R. A. (1970). Positive forgetting: The noninterference of items intentionally forgotten. Journal of Verbal Learning \& Verbal Behavior, 9, 255-268.

Bolte, A., Goschke, T., \& Kuhl, J. (2003). Emotion and intuition: Effects of positive and negative mood on implicit judgments of semantic coherence. Psychological Science, 14, 416-421.

Bradley, M. M., Cuthbert, B. N., \& Lang, P. J. (1996). Picture media and emotion: Effects of a sustained affective content. Psychophysiology, 33, 662-670.

Clore, G. L., \& Huntsinger, J. R. (2007). How emotions inform judgment and regulate thought. Trends in Cognitive Sciences, 11, 393-399.

Conway, M. A., Harries, K., Noyes, J., Racsma'ny, M., \& Frankish, C. R. (2000). The disruption and dissolution of directed forgetting: Inhibitory control of memory. Journal of Memory \& Language, 43, 409-430.

Corson, Y., \& Verrier, N. (2007). Emotions and false memories: Valence or arousal? Psychological Science, 18, 208-211.

Delaney, P. F., \& Sahakyan, L. (2007). Unexpected costs of high working memory capacity following directed forgetting and contextual change manipulations. Memory \& Cognition, 35, 1074-1082.

Duyck, W., Desmet, T., Verbeke, L. P. C., \& Brysbaert, M. (2004). WordGen: A tool for word selection and nonword generation in Dutch, English, German, and French. Behavior Research Methods, Instruments, \& Computers, 36, 488-499.

Eich, E., \& MetCalfe, J. (1989). Mood dependent memory for internal versus external events. Journal of Experimental Psychology: Learning, Memory, \& Cognition, 15, 443-455.

GARRETT, A. S., \& MADDOCK, R. J. (2001). Time course of the subjective emotional response to aversive pictures: Relevance to fMRI studies. Psychiatry Research: Neuroimaging, 108, 39-48.

Gasper, K., \& Clore, G. L. (2002). Attending to the big picture: Mood and global versus local processing of visual information. Psychological Science, 13, 34-40.

Geiselman, R. E., Bjork, R. A., \& Fishman, D. L. (1983). Disrupted retrieval in directed forgetting: A link with posthypnotic amnesia. Journal of Experimental Psychology: General, 112, 58-72.

HäNZE, M., \& Hesse, F. W. (1993). Emotional influences on semantic priming. Cognition \& Emotion, 7, 195-205.

Isen, A. M., Johnson, M. M. S., Mertz, E., \& Robinson, G. F. (1985). The influence of positive affect on the unusualness of word associations. Journal of Personality \& Social Psychology, 48, 1413-1426.

Lang, P. J., Bradley, M. M., \& CUThberT, B. N. (1999). International Affective Picture System (IAPS): Technical manual and affective ratings. Gainesville, FL: University of Florida, Center for Research in Psychophysiology.

MacLeod, C. M. (1998). Directed forgetting. In J. M. Golding \& C. M. MacLeod (Eds.), Intentional forgetting: Interdisciplinary approaches (pp. 1-57). Mahwah, NJ: Erlbaum.

McNally, R. J., Clancy, S. A., Barrett, H. M., \& Parker, H. A. (2004). Inhibiting retrieval of trauma cues in adults reporting histories of childhood sexual abuse. Cognition \& Emotion, 18, 479-493.

Mitchell, R. L. C., \& PhiLlips, L. H. (2007). The psychological, neurochemical and functional neuroanatomical mediators of the effects of positive and negative mood on executive functions. Neuropsychologia, 45, 617-629.

Moulds, M. L., \& BRYANT, R. A. (2005). An investigation of retrieval inhibition in acute stress disorder. Journal of Traumatic Stress, 18, 233-236.

Russell, J. A., Weiss, A., \& Mendelsohn, G. A. (1989). Affect Grid: A single-item scale of pleasure and arousal. Journal of Personality \& Social Psychology, 57, 493-502.

Sahakyan, L., \& Delaney, P. F. (2003). Can encoding differences explain the benefits of directed forgetting in the list method paradigm? Journal of Memory \& Language, 48, 195-206.

SAHAKYAN, L., \& GoOdMON, L. B. (2007). The influence of directional associations on directed forgetting and interference. Journal of Experimental Psychology: Learning, Memory, \& Cognition, 33, 1035-1049.

Sahakyan, L., \& Kelley, C. M. (2002). A contextual change account of the directed forgetting effect. Journal of Experimental Psychology: Learning, Memory, \& Cognition, 28, 1064-1072.

Schwarz, N., Bless, H., \& Bohner, G. (1991). Mood and persuasion: 
Affective states influence the processing of persuasive communications. In M. Zanna (Ed.), Advances in experimental social psychology (Vol. 24, pp. 161-199). New York: Academic Press.

Storbeck, J., \& Clore, G. L. (2005). With sadness comes accuracy; with happiness, false memory: Mood and the false memory effect. Psychological Science, 16, 785-791.

ZELlner, M., \& B ÄUmL, K.-H. (2006). Inhibitory deficits in older adults: List-method directed forgetting revisited. Journal of Experimental Psychology: Learning, Memory, \& Cognition, 32, 290-300.

\section{NOTE}

1. Within the IAPS, the emotion evoked by a photograph is assessed using Likert-type rating scales ranging, for the dimension of affective valence, from 1 (extremely negative) to 9 (extremely positive) and, for the dimension of emotional arousal, from 1 (low arousal) to 9 (high arousal).

(Manuscript received October 5, 2007; revision accepted for publication July 3, 2008.) 